\title{
Surface reflectance of Hintereisferner, Austria, from Landsat 5 TM imagery
}

\author{
Robert Koelemeijer, Johannes Oerlemans and Stephen TJemkes \\ Institute for Marine and Atmospheric Research, Princetonplein 5, 3584 CC Utrecht, The Netherlands
}

\begin{abstract}
Landsat 5 Thematic Mapper observations are used to derive the horizontal distribution of reflectance over Hintereisferner in Austria. To analyze temporal evolution of surface reflectance, three different dates are considered. The retrieval method adopts the linear relation between the planetary and surface reflectance, which follows directly from the principles of invariance. Constants in this linear relation are determined using a simple two-stream radiation model for each of the three days under consideration. From a comparison with observations the retrieved albedo is shown to compare favorably with ground observations.
\end{abstract}

\section{INTRODUCTION}

To make realistical estimation of glacier ablation by means of model simulations, the albedo over the entire glacier needs to be specified, for it is the albedo which determines to a large extent the energy balance and thus the ablation (Van de Wal and others, 1992). In general reflectance decreases from head to front. Detailed. distribution depends strongly on the physical state of the surface, especially the presence of crevasses, dust, moraine material and air bubbles in the ice. Unfortunately the ice surface is largely heterogenous, which makes extrapolation of in situ observations difficult, especially for large glaciated surfaces like subpolar ice caps and the Greenland ice sheet.

Space-borne observations of reflected solar radiation could in principle be used to monitor the spatial and temporal structure of the reflectance. The retrieval of surface reflectance from these observations has been a topic of active research (e.g. Dozier, 1989; Hall and others, 1989; Kaufmann, 1989). It is a priori not clear that existing algorithms which were especially derived for application over spatially homogeneous areas, can readily be applied to retrieve surface reflectance for glaciated surfaces.

In the present paper we report on the retrieval of the reflectance of Hintereisferner in Austria $\left(46^{\circ} 50^{\prime} \mathrm{N}\right.$, $10^{\circ} 50^{\prime} \mathrm{E}$ ) from space-borne observations of reflected solar radiation by the Landsat 5 Thematic Mapper. The objective of the present study is to analyse the spatial and temporal distribution of surface reflectance, without too much emphasis on the absolute value. Spatial and temporal evolution of surface reflectance has roughly the same pattern every year. This allows us to make a useful comparison between space-borne and in situ observations of the surface reflectance taken during different ablations seasons in at least a qualitative way. The in situ
Table 1. Landsat 5 Thematic Mapper spectral data

Channel

Spectral interval

$\mu \mathrm{m}$
$0.45-0.52$
$0.52-0.60$
$0.63-0.69$
$0.76-0.90$
$1.55-1.75$
$2.08-2.35$

observations were obtained during a glacio-meteorological experiment on Hintereisferner in summer 1989, (Van de Wal and others, 1992). These ground observations could in principle be used to validate the retrieved reflectance, except that no temporal collocated Landsat observations exist.

Three Landsat 5 Thematic Mapper scenes were used to study the evolution of reflectance, one at the beginning of the ablation season on 4 May 1989, one in the middle on 20 July 1988 and one at the end on 22 September 1988. Here we consider observations in the visible (channels 1-4) and near-infrared (channels 5 and 7) channels (Table 1). The Thematic Mapper has a horizontal resolution of $30 \mathrm{~m}$.

To determine surface reflectance from these observations, the latter must be corrected for the interaction between radiation and the atmospheric constituents. This is done using the linear relations between the planetary 
and surface reflectance, which follow directly from the principles of invariance (see below: "Retrieval method"). The proportionality constants in this relation are dependent upon the particular spectral interval, the specific thermodynamical profiles and solar zenith angle, and constants are determined from a simple two-stream radiative transfer model. The total reflectance is found as a linear combination of the channels 2, 4 and 7, or the channels 4 and 7 spectral reflectance, depending on the saturation of channel 2. Results for the three days are discussed in "Results" below.

\section{RETRIEVAL METHOD}

Consider a spectral interval in the shortwave part of the spectrum. According to the principles of invariance (Chandrashekhar, 1960), which in essence is a statement about the linearity of the radiation field, the upwelling shortwave flux at the top of the atmosphere is the linear combination of the downwelling flux at the top of the atmosphere reflected by the total atmospheric column and the upwelling flux from the surface transmitted through this column:

$$
F_{\text {toa }}^{+}=R F_{\text {toa }}^{-}+T F_{\mathrm{sfc}^{2}}^{+}
$$

where $F$ represents the flux $\left(\mathrm{W} \mathrm{m}^{-2}\right)$ in the upward $\left(_{+}\right.$ superscript) or downward (- superscript) at the top of atmosphere (toa subscript) or surface (sfc subscript). Atmospheric reflectance and transmission are represented by $R$ and $T$ respectively. Replacing the upwelling flux at the surface by the product of the surface reflectance and downwelling surface flux $F_{\mathrm{sfc}}^{+}=R_{\mathrm{sfc}} F_{\mathrm{sfc}}^{-}$we find the linear relation between the surface and the planetary reflectance $\left(R_{\text {toa }}=F_{\text {toa }}^{+} / F_{\text {toa }}^{-}\right)$, which after some trivial manipulation can be written as

$$
R_{\mathrm{toa}}=a+b R_{\mathrm{sfc}}
$$

This form was used also by Koepke (1989) in his study of the retrival of surface reflectance of vegetated areas from Advanced Very High Resolution Radiometer (AVHRR) observations. It is clear that to derive $R_{\mathrm{sfc}}$ from the space-borne observation of the reflected solar radiation, the constants $a$ and $b$ in Equation (2) need to be specified. These depend upon the particular spectral interval and actual atmospheric thermal structure. They are determined using a two-stream radiative transfer model.

In the radiation model employed in the present study, the effects of Rayleigh scattering as well as absorption by ozone and water vapour on the transfer of shortwave radiation through the atmosphere are parameterized. The gaseous absorption by water vapour and ozone is modeled using the sum of exponential method (Stephens, 1984). In this method the transmission in a spectral band of a homogeneous layer is given by

$$
T(u)=\sum_{i=1}^{n} \beta_{i} e^{-\gamma_{i} u},
$$

in which $u$ represents the absorber amount. Simple pressure and temperature scaling is used to simulate the transfer through inhomogeneous layers (Stephens, 1984).
Table 2. Constants $a$ and $b$ in the linear relation between the planetary and surface reflectance as derived from model calculations for each of the 6 spectral intervals considered here. The calculations were done for a standard midlatitude summer profile (McClatchey and others, 1971) which is assumed to be representative for the three days. Solar inclination is taken from Landsat CCT tapes

Channel 4 May 198

20 July 198822 September 1988

$\begin{array}{llllll}a & b & a & b & a & b\end{array}$

\begin{tabular}{lllllll}
\hline & & & & & & \\
1 & 0.049 & 0.882 & 0.046 & 0.885 & 0.066 & 0.863 \\
2 & 0.021 & 0.892 & 0.020 & 0.894 & 0.030 & 0.877 \\
3 & 0.009 & 0.910 & 0.009 & 0.912 & 0.014 & 0.899 \\
4 & 0.007 & 0.926 & 0.006 & 0.927 & 0.008 & 0.921 \\
5 & 0.001 & 0.837 & 0.001 & 0.837 & 0.001 & 0.834 \\
6 & 0.001 & 0.819 & 0.001 & 0.820 & 0.001 & 0.814 \\
\hline
\end{tabular}

The constants $\beta_{i}$ and $\gamma_{i}$ are adopted from Slingo and Schrecker (1982).

In Table 2 values of $a$ and $b$ for the six spectral intervals considered and the three days are presented. A standard midlatitude summer profile (McClatchey and others, 1971) is assumed to be representative for the actual atmospheric thermal structure. The solar inclination is taken from the Landsat CCT tapes which allow for the glacier to be located at the center of the images. From test calculations it was concluded that $a$ and $b$ are insensitive to the actual water vapour content, mainly because of the mean surface elevation of $3000 \mathrm{~m}$ for the Hintereisferner. Note that $a$ and $b$ in Equation (2) do not include an atmospheric aerosol effect. As mentioned above, we are more interested in the horizontal distribution of surface reflectance than its absolute value. As long as the atmospheric aerosols fluctuate on a much larger characteristic length scale than surface reflectance, the aerosol effect in our retrieval method can be ignored.

While the satellite makes observations of specific intensity ( $\mathrm{I}$, the flow of energy from a particular direction units: $\left.\mathrm{W} \mathrm{m}^{-2} \mathrm{sr}^{-1}\right)$, the radiation model simulates the total flux (the flow of energy through a horizontal plane units: $\mathrm{W} \mathrm{m}^{-2}$ ). By definition, the upward flux is the first moment of the specific intensity over the upper half hemisphere (Chandrasekhar, 1960):

$$
F^{+}=2 \pi \int_{0}^{1} I(\mu) \mu \mathrm{d} \mu,
$$

with $\mu$ the cosine of the angle between the normal and propagation direction. This equation can be solved only when the angular dependence of the specific intensity is specified. Here we assume that the observed upwelling radiation field is isotropic, $I \neq I(\mu)$, such that Equation (4) can readily be evaluated to yield:

$$
F_{\text {obs }}^{+}=\pi I_{\text {obs }} \text {. }
$$


The spectral planetary reflectance defined by the ratio of the upwelling and downwelling flux at the top of the atmosphere follows directly

$$
R_{\text {toa }} \equiv \frac{F_{\text {toa }}^{+}}{F_{\text {toa }}^{-}}=\frac{\pi I_{\text {obs }}}{S_{\odot} \cos \left(\theta_{\odot}\right)},
$$

with $S_{\odot}$ the solar insolation at the top of the atmosphere. Finally, to convert the spectral to a spectral mean surface reflectance, the simple relationship proposed by Duguay and LeDrew (1991) was used. In the case that channel 2 was not saturated, they proposed a 3-channel relation

$$
R_{\mathrm{sfc}}=0.526 R_{2}+0.204 R_{4}+0.015 R_{7} .
$$

In the case that channel 2 was saturated they used a 2channel relation

$$
R_{\mathrm{sfc}}=0.793 R_{4}+0.015 R_{7}
$$

Horizontal distribution of retrieved reflectance for the different days is presented in Figure 1. In the upper left corner a geographical map of the lower part of the Hintereisferner is presented on roughly the same scale. Clearly shown is the large horizontal variation of reflectance over the glacier. Even in May, when the glacier is still covered by snow, the horizontal distribution of reflectance is not entirely homogenous. Some small
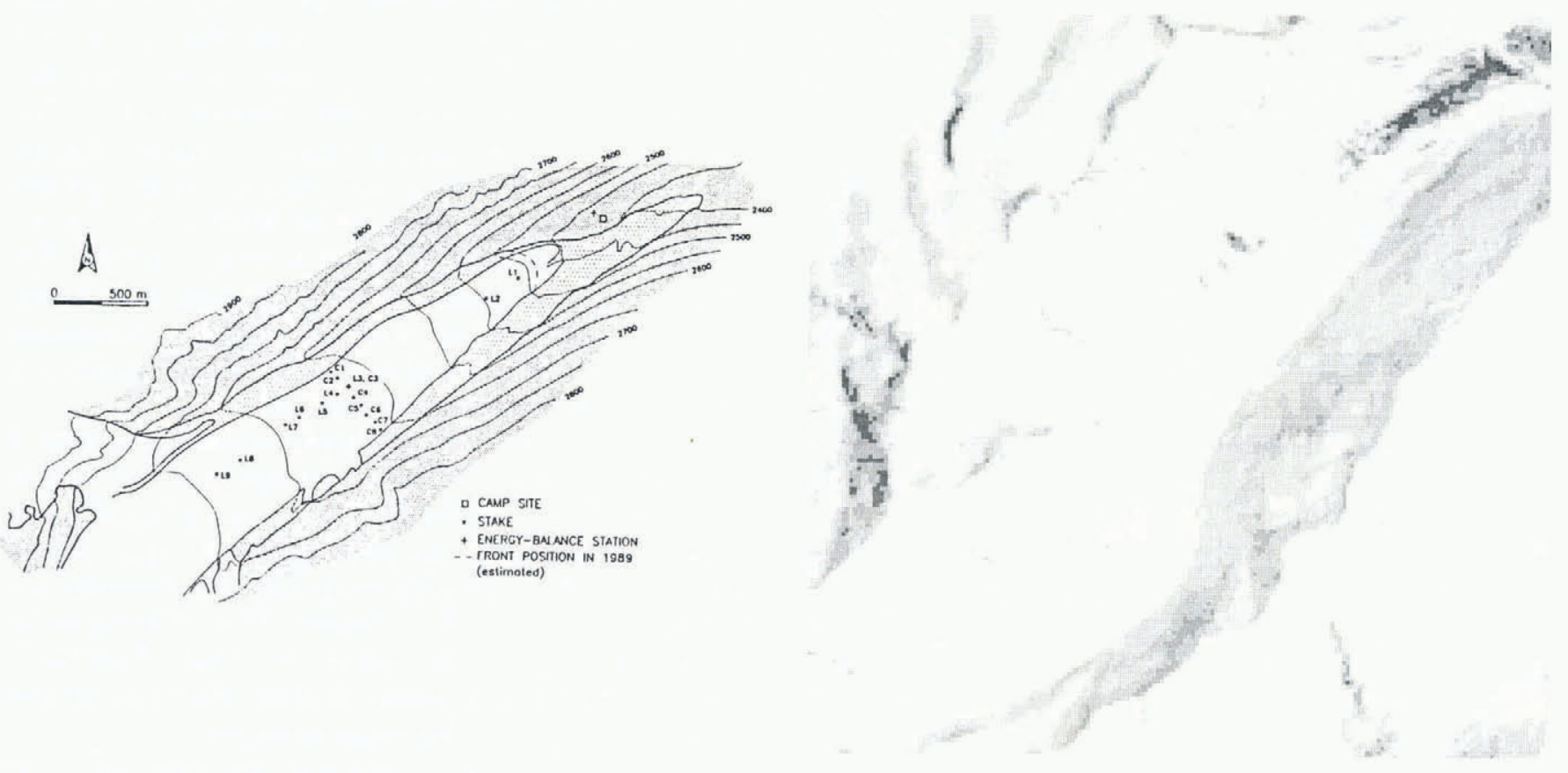

a

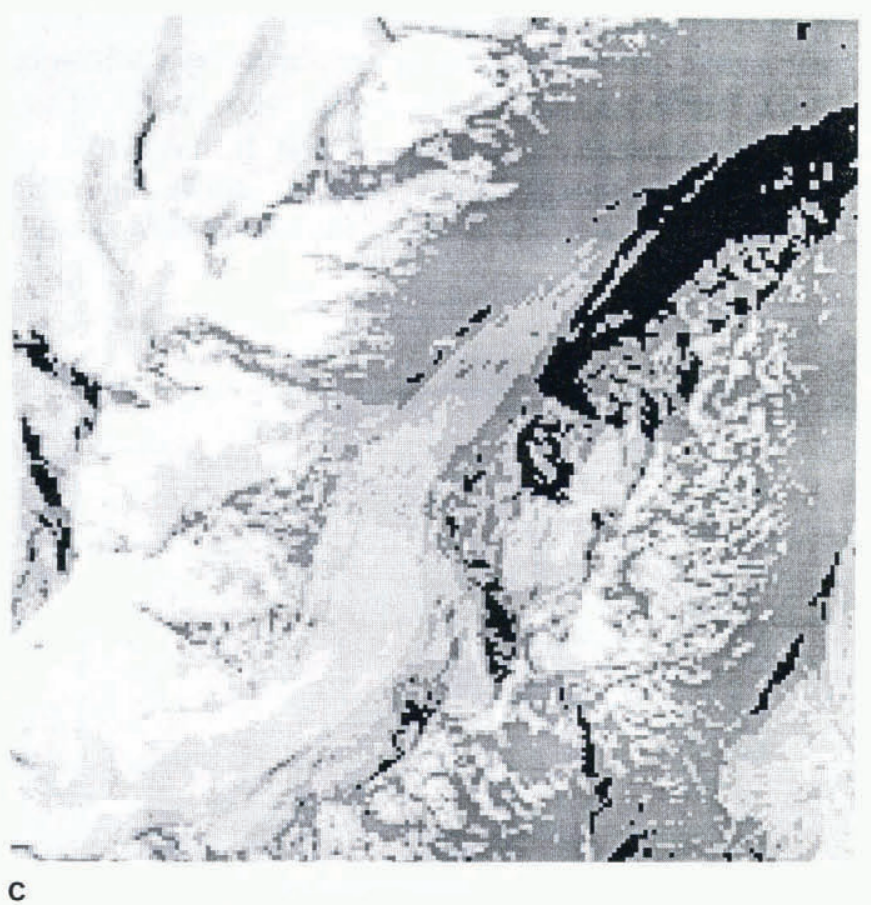

b

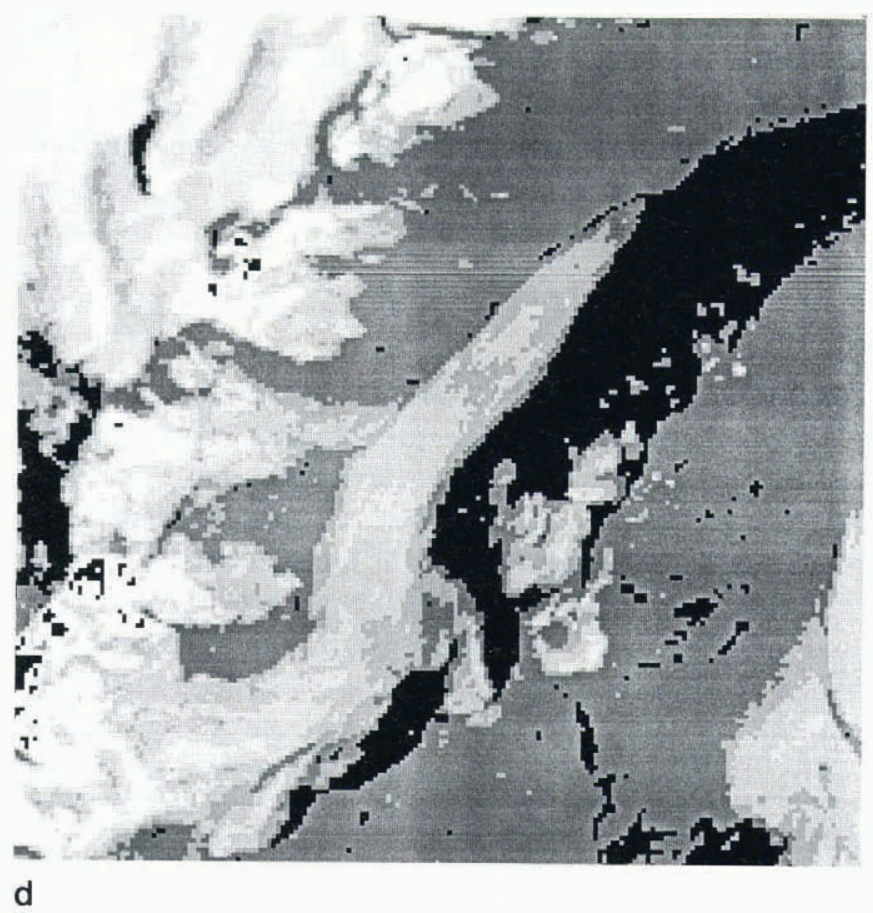

Fig. 1. Horizontal distribution of the total reflectance of Hintereisferner for three different stages of the ablation season, (a) geographical map of the area at the same scale, (b) at the beginning (4 May, 1989), (c) middle (20 July 1988), (d) at the end (22 September 1988). 

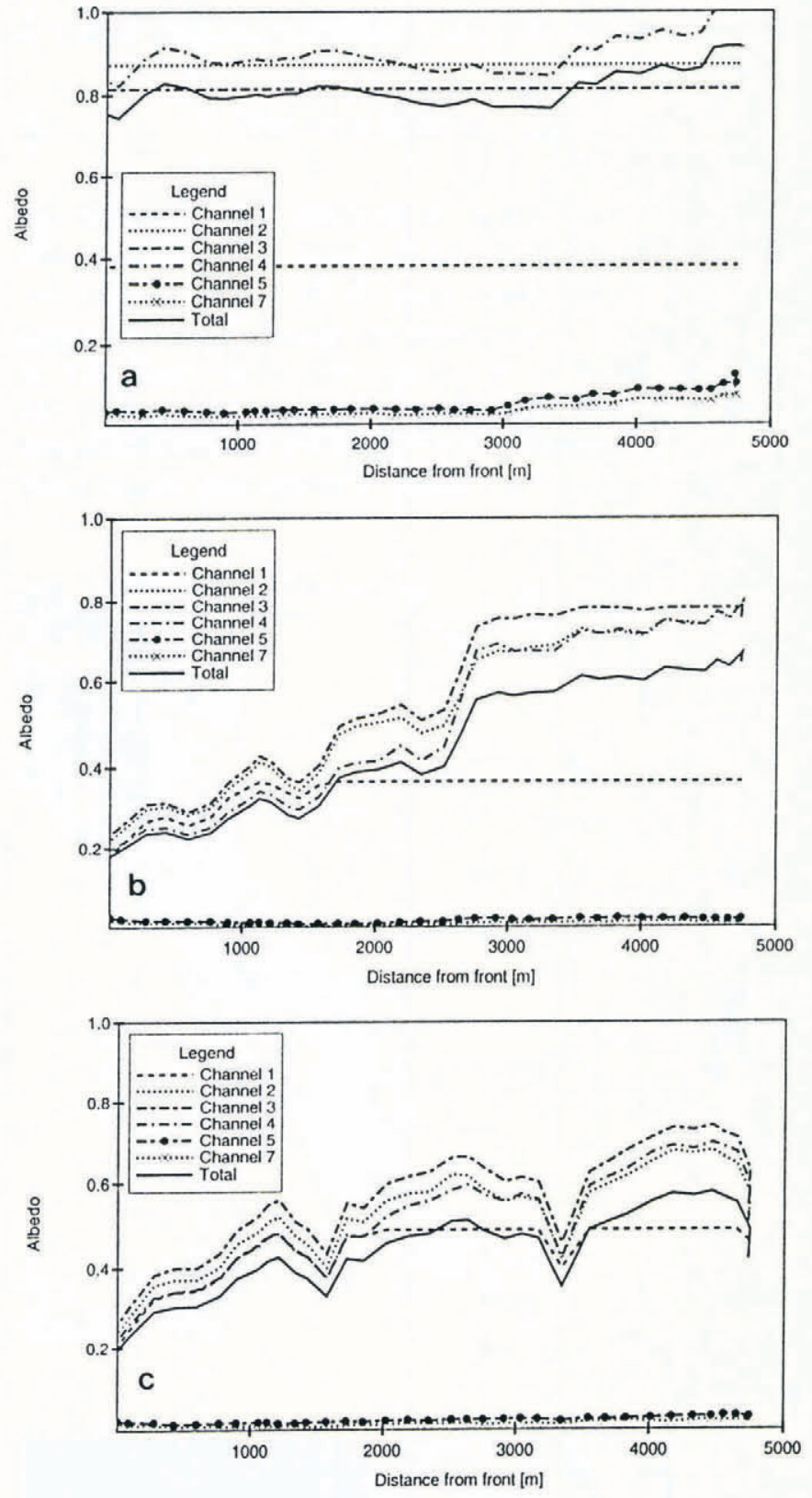

Fig. 2. Total and spectral reflectance along a longitudinal path over the glacier. (a) 4 May 1989, (b) 20 July 1988, (c) 22 September 1988 .

regions with a somewhat lower reflectance are visible, probably due to the presence of crevasses. In the middle and at the end of the season more details are present than at the beginning, as a result of the heterogeneous structure of the physical state of the glacier surface, i.e. dust and air bubble concentration, presence of crevasses and moraine material. While air bubble concentration tends to increase the reflectance, dust, crevasses and moraine material tend to decrease reflectance (cf. Dozier, 1989; Mullen and Warren, 1988; Pfeffer and Bretherton, 1987; Warren and Wiscombe, 1980; Wiscombe and Warren, 1980).

In Figure 2 we present the retrieved albedo along a longitudinal profile of Hintereisferner for the three days. This path is collated with the position of stakes L1-L9 used in the field campaign described below. Each point is the average of nine adjacent points. This is done to reduce the variability introduced by local inhomogeneities, so that a better representation of the general profile is obtained. We show spectral as well as total reflectance as a function of the approximate distance from the front. In May, the glacier is still totally covered by snow at the beginning of the ablation season. Total reflectance increases from 0.75 at the front to 0.90 at the head, probably due to a decrease of grain size. This can be clearly seen in the near-infrared, which is sensitive to grain size (Wiscombe and Warren, 1980).

In July, at $2500 \mathrm{~m}$ from the front, reflectance suddenly increases by almost 0.20 . this sudden increase is associated with a rapid change of surface texture. While above this line the glacier is still covered by snow, below it all snow has been melted. Spectral reflectance in channels 2, 3 and 4 (Fig. 2) shows roughly the same pattern as total reflectance. These channels are sensitive to impurities (Warren and Wiscombe, 1980; Dozier, 1989). The general decreasing reflectance down the glacier may be partly due to the increasing dust concentration. Spectral reflectance is also influenced by the presence of crevasses and air bubble concentration. It is to be expected that dips in reflectance around 700, 1500 and $2300 \mathrm{~m}$ are caused by these crevasses. Lack of additional information about the physical state of the glacier surface prohibits a detailed explanation of these features.

At the end of the ablation season no sudden increase of the reflectance associated with a change in surface texture is present (Fig. 2). Reflectance increases rapidly the first $1000 \mathrm{~m}$ or so, but after that remains fairly constant. Again some areas where the reflectance suddenly decreases are visible, possibly due to crevasses. The reflectances in channels 2, 3 and 4 show a similar behaviour. Differences between spectral reflectances in these channels are less than at the middle of the ablation season.

In Figure 3 the total reflectance for three days as a function of the distance to the glacier front is presented. In the middle of the ablation season, reflectance is less than at the beginning of the season. While the difference between reflectance at the beginning and middle of the ablation season at the glacier head is only 0.25 , it increases to 0.60 at the front. Near the glacier head the reflectance is lower at the end of the season than at mid-

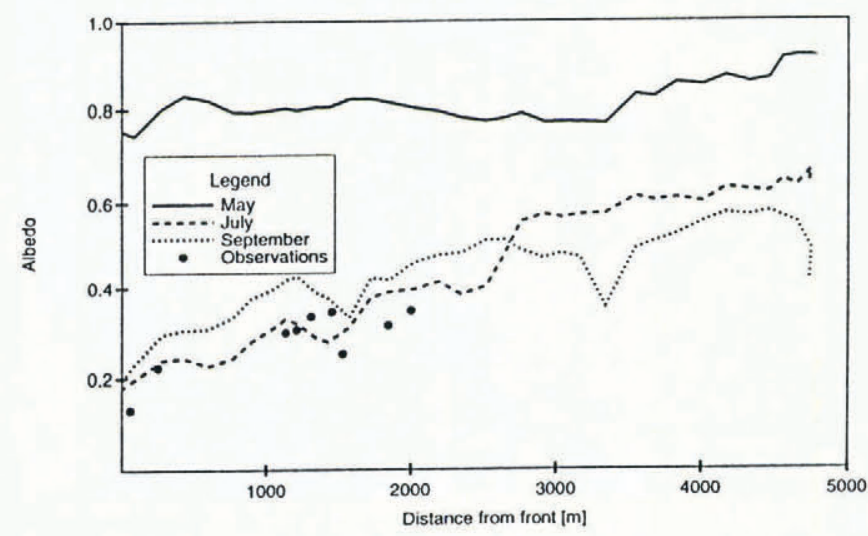

Fig.3. Total albedo along a longitudinal path over the glacier for the three different days. Also shown are the ground observations taken during a field experiment in 1989, adopted from Van de Wal and others (1992). 
season, while near the front the reverse is true. This is a common feature; a glacier appears to be somewhat brighter towards the end of the ablation season than in the middle, possibly due to the larger value of the solar zenith angle. Scattering of visible radiation by the air bubbles in the ice is mainly in the forward direction. This implies that the chance for a photon to scatter out of the ice increases with increasing solar zenith angle.

Also shown in this figure are the in situ measurements averaged over the period 13 July to 16 September 1989 along a longitudinal path on the glacier obtained during the 1989 field campaign (Van de Wal and others, 1992) at the positions L1-L9 shown on the geographical map. The reflectance at $1.5 \mathrm{~m}$ above the surface was measured with a hand-held reflectionmeter, which compared with a standard Aandera pyranoradiometer has an absolute accuracy of about 2\% (Van de Wal and others, 1992). Thus uncertainties in the absolute value of the observed reflectance are much smaller than the horizontal variation. Although the retrieved reflectance appeared to be low for a glacier, it compares favorably with the in situ observations, which indicate that at the snout the reflectance is even lower than values retrieved from the Landsat 5 observations. This may be the result of the steep surface slope there. In the present method the reflectance for a horizontal plane is retrieved, while the observations were taken parallel to the surface. Since Hintereisferner has an average slope of a few per cent, no large differences were observed during the observational campaign between the reflectance parallel to the surface and the horizontal plane reflectance.

The cross profile of total reflectance (Fig. 4) shows a decrease of reflectance from the middle of the glacier to the sides. When compared with the in situ measurements obtained at stake positions $\mathrm{Cl}-\mathrm{C} 8$ on the map, again a good correspondance is found.

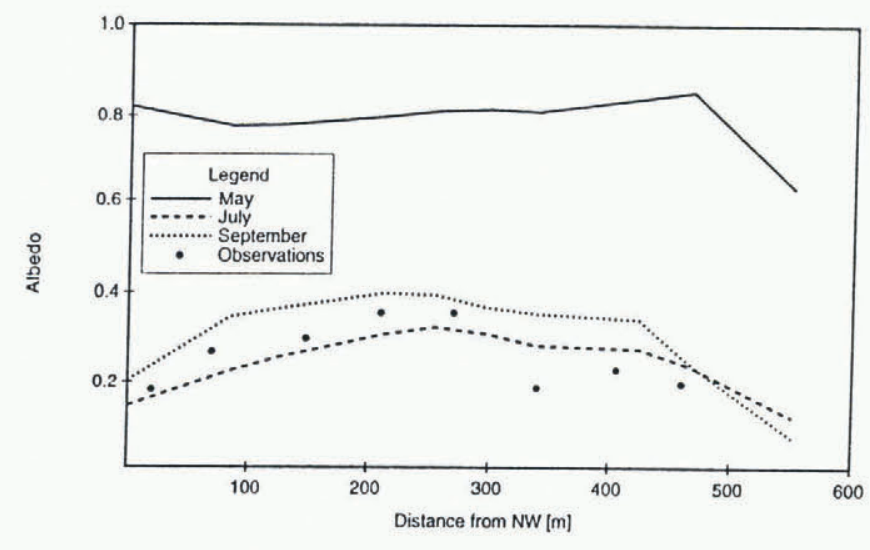

Fig. 4. Total reflectance along a cross-glacier path for the three different days. Also shown are ground observations taken during a field experiment in 1989, adopted from Van de Wal and others (1992).

\section{SUMMARY}

The horizontal distribution of surface reflectance over Hintereisferner has been derived from Landsat 5 Thematic Mapper observations at different times in the ablation season (May, 1989; July, 1988; September, 1988). Ground observations of surface reflectance taken during a field campaign in 1989 have been adopted as a reference for the retrieved albedo. Although no temporal correlation exists between the spaceborne and in situ measurements, it has been shown that retrieved reflectance compares favorably with in situ measurements. This is quite surprising, given the dependence of reflectance upon the physical state of the ice and various simplifications made in the retrieval method. It is quite possible that the good correlation is the result of error cancellation. For instance, the linear relation adopted to relate the spectral reflectances to the total reflectance is derived for snow-covered terrain, while here we used it for glacier ice. The spectral characteristics of glacier ice differ from that of snow, especially when this ice contains dust and liquid water. It is possible that a different weighting formula more appropriate for glacier ice containing dust and liquid water yields lower values. The error introduced by the weighting formula is probably offset by the error in the hemispheric mean reflectance introduced by assuming that the glacier reflects isotropically. For the adopted conversion of the directional reflectance, observed at an angle different from the specular, to hemispheric mean reflectance, results in an underestimation of the actual hemispheric mean reflectance. It is clear that a proper accuracy assessment of the adopted retrieval method can be made only from detailed observations of all relevant parameters comparable with Landsat 5 observations.

\section{ACKNOWLEDGEMENT}

Financial support for this project was provided by the Dutch foundation for space research (SRON).

\section{REFERENCES}

Chandrasekhar, S. 1960. Radiative transfer. New York, Dover Publications.

Dozier, J. 1989. Spectral signature of alpine snow cover from the Landsat Thematic Mapper. Remote Sensing Environ., 28, 9-22.

Duguay, C. and E. LeDrew. 1991. Mapping surface albedo in the east slope of the Colorado Front Range, U.S.A. with Landsat Thematic Mapper. Arct. Alp. Res., 23, 213-223.

Hall, D. K., A. T. C. Chang, J. L. Foster, C. S. Benson and W. M. Kovalick. 1989. Comparison of in situ and Landsat derived reflectance of Alaskan glacier. Remote Sensing Environ., 28, 23-31.

Kaufman, Y. 1989. The atmospheric effect on remote sensing and its correction. In Asrar, G. ed.Theory and applications of optical remote sensing. New York, John Wiley and Sons, 336-428.

Koepke, P. 1989. Removal of atmospheric effects from AVHRR reflectances. 7. Appl. Meteorol., 28, 13411348.

McClatchey, R., R. Fenn, J. Selby, F. E. Volz and L. Garing. 1971. Optical properties of the atmosphere. Air Force Geophysical Laboratory, Hanscom AFB, MA. AFGL Rep. 71-0279. 
Mullen, P. C. and S. G. Warren. 1988. Theory of the optical properties of lake ice. J. Geophys. Res., 93(D7), 8403-8414.

Pfeffer, W. T. and C. S. Bretherton. 1987. The effect of crevasses on the solar heating of a glacier surface. International Association of Hydrological Sciences Publication 170 (Symposium at Vancouver 1987 - The Physical Basis of Ice Sheet Modelling), 191-205.

Slingo, A. and H. Schrecker. 1982. On the shortwave radiative properties of stratiform water clouds. Q. J.R. Meteorol. Soc., 108, 407-426.

Stephens, G. 1984. The parameterization of radiation for numerical weather prediction and climate models. Mon. Weather Rev., 112, 826-867.
Wal, R. S. W. van de, J. Oerlemans and J. van der Hage. 1992. A study of ablation variations on the tongue of Hintereisferner, Austrian Alps. F. Glaciol. 38(130), 319-324

Warren, S. G. and W. J. Wiscombe. 1980. A model for the spectral albedo of snow. II. Snow containing atmospheric aerosols. F. Atmos. Sci., 37(12), 2734-2745.

Wiscombe, W.J. and S. G. Warren. 1980. A model for the spectral albedo of snow. I. Pure snow. F. Atmos. Sci., 37(12), 2712-2733.

The accuracy of references in the text and in this list is the responsibility of the authors, to whom queries should be addressed. 